\title{
Adaptive Approximate Dynamic Optimization : a slider-crank case study
}

\author{
Tom Lefebvre ${ }^{1,2, *}$, Frank Naets ${ }^{3,4}$, Frederik De Belie ${ }^{1,2}$, Guillaume Crevecoeur ${ }^{1,2}$
}

\begin{abstract}
We present the Adaptive Approximate Dynamic Optimization (AADO) algorithm and elaborate on its application for forward (dynamics as constraints) and inverse (dynamics in the objective) Dynamic Optimization (DO). The method is tailored to dynamic optimization problems where the system model contains high-fidelity (HF) model components of subsystem(s) that slow down the system model's evaluation time. In this contribution we extend prior work where we proposed to replace the system model with a local (i.e. only valid in subregions of the state space) approximate dynamic system model based on sampled model evaluations. The sampling is adaptive and localized to the optimal trajectory in correspondence with the trajectory optimizer. Here we suggest only to replace the high-fidelity subsystem models with an approximate subsystem model set. A comparison is made of the method applied in both the forward and inverse convention on a limit cycle slider-crank trajectory optimization for a high-fidelity nonlinear load model. We found that the AADO algorithm requires only $0.1 \%$ system model evaluations compared with conventional DO and only $30 \%$ compared with non-adaptive AADO. Our results consolidate the methods potential and invite for further research.
\end{abstract}

\section{INTRODUCTION}

An ubiquitous task in mechatronics is to develop systems that exhibit optimal behaviour. Reduction in energy consumption, minimal execution time, increased stability or succesfull execution of a highlevel goal, are typical goals in mechatronic system design. A popular framework to address this type of problems is the framework of Dynamic Optimization which requires a dynamic system model. It is key to assure close correspondence between the optimized and the actual system. High-Fidelity (HF) dynamic models can contain multidomain physics and intricate subsystems interactions, slowing down the model's evaluation time.

Numerical solution of such Dynamic Optimization Problems (DOP) is often facilitated by a class of so-called direct methods, among which direct multiple shooting (DMS) is the most widely applied. An emergent problem when a HF dynamic model is coupled to a (direct) trajectory optimizer, is that a large amount of function evaluations will be called for by the optimizer. E.g. for the case study discussed in section IV] a single limit cycle optimization required about 30, 000 dynamic model functions evaluations (gradients were computed using finite differencing which will be required given the assumptions made with respect to the HF model).

In [1] we proposed a method to deal with this problem that is inspired on metamodel based optimization methods. We suggested to replace the HF model with an approximator that is fitted on sampled HF model evaluations. Previously we approached the problem by approximating the system level model. Here we abstain from this idea and propose only to

\footnotetext{
${ }^{1}$ Department of Electrical Energy, Metals, Mechanical Constructions \& Systems, Ghent University, B-9052 Ghent, Belgium.

${ }^{2}$ EEDT Decision and Control, Flanders Make.

${ }^{3}$ Department of Mechanical Engineering, Katholieke Universiteit Leuven, Leuven, Belgium

${ }^{4}$ DMMS lab, Flanders Make, Lommel, Belgium

*Corresponding author: tom. lefebvre@ugent.be.
}

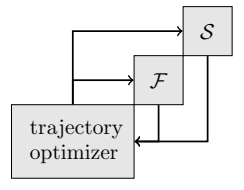

(a) using high-fidelity model components

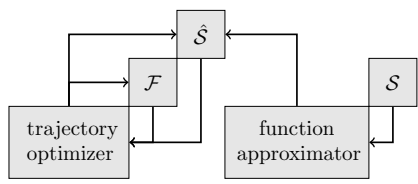

(b) using approximate dynamics

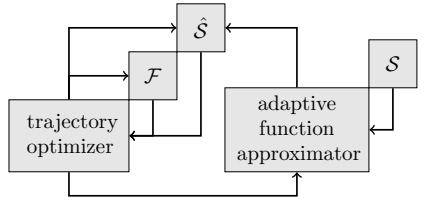

(c) adaptive approximate dynamics

Fig. 1: Several possible approaches for trajectory optimization with high-fidelity model component set, $\mathcal{S}$.

approximate a subset of HF subsystems provided that such a classification can be made and that the subsystems can be evaluated seperately.

In this context, such an approximate model can be constructed from a number of high-fidelity model component samples using any function approximation method [5], [6]. Such a sample set can be gathered offline, before coupling the approximate model to the optimizer using state-of-the-art adaptive global sampling algorithms, e.g. [7], [8].

The problem with this strategy is that a globally valid approximation model will be obtained that accuratly emulates the actual physical model over the entire feasible model region. To accomplish this feature a large number of samples will be collected. That, whilst the eventual optimal trajectory will only cover a small subregion of the feasible area, and the sample effort could therefore be concentrated to this subregion. The basic insight of the AADO algorithm is to use the optimizer as guidance to focus all sample effort to the relevant feasible subregion where the solution is located.

This idea is formalized in Fig 1 . Here all fast model components (really any analytical model component describing interactions and physics first principles) are combined in the set, $\mathcal{F}$, whilst all slow model components (modelling intecrate processes that can not be formulized, e.g. finite element models) are combined in a set, $\mathcal{S}$.

We proceed as follows. In section II we provide a general overview of the problem for a broad class of mechanical dynamic system models. In section [III] we discuss the AADO algorithm and address the question on how to generate samples in the neighbourhood of a temporary (possibly unconverged) trajectory. We present simulations results for a slider-crank case study in section IV

\section{PRELIMINARIES}

\section{A. A general framework for mechanical dynamic modeling}

We first describe a class of mechanical dynamic system models for which the AADO algorithm may be useful. 
Regarding general rigid (multi)body systems, the NewtonEuler equations of motion can be arranged into the following form, which can be engaged consecutively to describe the dynamic behaviour of the $n$ bodies being considered

$$
\mathrm{A} \ddot{\mathbf{p}}=\mathrm{Br}+\mathrm{C} \boldsymbol{\tau}+\mathrm{Dw}
$$

We reserve $A$ to denote a general inertia matrix gathering all the system body's lumped masses and moments of inertia. The matrices $\mathrm{B}, \mathrm{C}$ and $\mathrm{D}$ are position dependent projection matrices that map respectively the contraint forces $\mathbf{r}$, the control forces $\tau$ and any active interaction forces $\mathbf{w}$, on their contribution to the total net forces and torques, that act on the center of mass (com) of each individual body. The force $\mathbf{w}$ is used to include any interaction with the environment that can not be modelled using prescribed framework.

We have expressed the general system description in function of cartesian coordinates $\mathbf{p}$. However given knowledge of an explicit forward kinematic function, $\mathbf{p}=\mathbf{h}(\mathbf{q})$, expressing the constraints (as are determined by the rigid connections between the $n$ bodies) in function of a set of generalised coordinates $\mathbf{q}$, this system can hence be transformated to the explicitly constrained system given below, where $J$ represents the jacobian of the forward kinematic model, $\mathbf{h}$.

$$
\mathrm{AJ} \dot{\mathbf{v}}+\mathrm{A} \dot{\mathbf{J}} \dot{\mathbf{q}}=\mathrm{Br}+\mathrm{C} \boldsymbol{\tau}+\mathrm{Dw}
$$

The latter is linear in function of $\dot{\mathbf{v}}, \boldsymbol{\tau}$ and $\mathbf{f}$ and hence we can solve for the couple $\{\dot{\mathbf{v}}, \mathbf{f}\}$ be it for the couple $\{\boldsymbol{\tau}, \mathbf{f}\}$. This will either generate the so called forward, $\ddot{\mathbf{q}}(\mathbf{q}, \dot{\mathbf{q}}, \mathbf{u})$, be it inverse, $\mathbf{u}(\mathbf{q}, \dot{\mathbf{q}}, \ddot{\mathbf{q}})$, dynamic model representations 1 . Note that this only holds when $\operatorname{dim}(\mathbf{u})=\operatorname{dim}(\mathbf{q})$ and when interaction term, $\mathbf{w}$, is independent of $\mathbf{f}$ and $\dot{\mathbf{v}}$ or $\tau$, which we will assume for the remainder of this article, i.e. $\mathbf{w}(\mathbf{q}, \dot{\mathbf{q}})$.

$$
\begin{gathered}
{\left[\begin{array}{ll}
\mathrm{AJ} & -\mathrm{B}
\end{array}\right] \cdot\left[\begin{array}{c}
\dot{\mathbf{v}} \\
\mathbf{r}
\end{array}\right]=\mathrm{Cu}-\mathrm{A} \dot{\mathrm{J}} \dot{\mathbf{q}}+\mathrm{Dw} \rightarrow \dot{\mathbf{v}}=\ddot{\mathbf{q}}(\mathbf{q}, \dot{\mathbf{q}}, \mathbf{u})} \\
{\left[\begin{array}{ll}
\mathrm{C} & \mathrm{B}
\end{array}\right] \cdot\left[\begin{array}{c}
\boldsymbol{\tau} \\
\mathbf{r}
\end{array}\right]=\mathrm{AJ} \ddot{\mathbf{q}}+\mathrm{AJ} \dot{\mathbf{q}}-\mathrm{D} \mathbf{w} \rightarrow \boldsymbol{\tau}=\mathbf{u}(\mathbf{q}, \dot{\mathbf{q}}, \ddot{\mathbf{q}})}
\end{gathered}
$$

In either case, the dynamic system description is an explicit function of force term, w, stemming from interaction with the environment. In the present work, our objective is to perform efficient trajectory optimization, in the specific case that interaction force term, w, poses a bottleneck for efficient (fast) numerical calculation [2]-[4].

\section{B. Forward and inverse numerical trajectory optimization}

Before we venture into detailing the AADO algorithm, we will first define a class of dynamic optimization problems to which it may be applied. For one to establish notation and more in particular to point out a crucial conceptual difference that affects application of the AADO algorithm.

$$
\begin{aligned}
\left\{\mathbf{x}^{*}, \mathbf{y}^{*}\right\}=\arg \min _{\mathbf{x}, \mathbf{y}} J[\mathbf{x}, \mathbf{y}] & =c\left(t_{f}\right) \\
\text { s.t. } \mathbf{h}\left(\mathbf{x}\left(t_{0}\right), \mathbf{x}\left(t_{f}\right)\right) & =0 \\
\mathbf{g}(\mathbf{x}, \mathbf{y}) & \leq 0, t \in \mathcal{T}=[0, T]
\end{aligned}
$$

\footnotetext{
${ }^{1}$ In this manuscript, we respect following definitions. Variables $\ddot{\mathbf{q}}$ and $\dot{\mathbf{v}}$ are reserved to denote system accelerations, here $\ddot{\mathbf{q}}$ is considered an optimization variable whilst $\dot{\mathbf{v}}$ is reserved to denote the physical change in velocity (both in generalized coordinate space). Variables $\mathbf{u}$ and $\tau$, on the other hand, are reserved to denote actuation forces, here $\mathbf{u}$ is considered an optimization variable whilst $\tau$ is reserved to denote the actual physical value. This convention proves to be usefull when the distinction between forward dynamic based and inverse dynamics based trajectory optimization should be emphasized.
}

Here we define system state, $\mathbf{x}=(\mathbf{q}, \dot{\mathbf{q}})$. Evolution of the system is predicted using a state-space model, $\mathbf{f}$, i.e. $\dot{\mathbf{x}}=\mathbf{f}(\mathbf{x}, \mathbf{y})$, as a function of $\mathbf{x}$ and a (yet unspecified) independent variable $\mathbf{y}$. Optimality of the trajectory is quantified through a cost, $c$. We assume that the cost accumulates over the trajectory and hence may be expressed through a scalar cost rate function, $l$, i.e. $\dot{c}=l(\mathbf{x}, \mathbf{y})$. We do not define a terminal penalty term as we will consider hard constraints, $\mathbf{h}$, that are active on the initial and final states, $\mathbf{x}_{0}$ and $\mathbf{x}_{f}$. Conclusively we can consider soft constraints $\mathrm{g}$ that apply over the entire time interval $\mathcal{T}$.

This is a well-studied problem. Numerical solution of (3) is performed most efficiently using a direct method [9], [10] that transcribes the continuous problem into a nonlinear program (NLP) that we can then solve using a nonlinear programmer, e.g. SQP. We use here the Direct Multiple Shooting (DMS) transcription method that approaches the system dynamics as hard constraints [9]. It has the advantage that continuous time signals, $\{\mathbf{x}, \mathbf{y}\}$, are parameterized through a finite number of shooting nodes, $\mathbf{X}=\left\{\mathbf{x}_{0}, \ldots, \mathbf{x}_{N}\right\}$ and $\mathbf{Y}=\left\{\mathbf{y}_{0}, \ldots, \mathbf{y}_{N}\right\}$, corresponding snapshots taken along the trajectories over $a(n)$ (equally spaced) time grid with discretisation $\mathbf{t}=\left\{t_{k} \mid t_{k+1}=t_{k}+\Delta\right\}_{k=0}^{N}$. As a result, the set of admissable trajectories is not limited to a subset corresponding any parametrization method and the fact that the optimization variables $\{\mathbf{X}, \mathbf{Y}\}$ affect the trajectories only locally will translate into a high problem sparsity.

Central to this transcription is the propagation operator $\mathcal{P}_{k}$ that executes local integration $\mathcal{P}_{k} \mathbf{a}(\mathbf{x}, \mathbf{y})=\int_{t_{k}}^{t_{k+1}} \mathbf{a}(\mathbf{x}, \mathbf{y}) \mathrm{d} t$ numerically as a function of $\{\mathbf{X}, \mathbf{Y}\}$, e.g. the trapezoidal rule $\mathcal{P}_{k} \mathbf{f}(\mathbf{x}, \mathbf{y})=\frac{\Delta}{2} \mathbf{f}\left(\mathbf{x}_{k}, \mathbf{y}_{k}\right)+\frac{\Delta}{2} \mathbf{f}\left(\mathbf{x}_{k+1}, \mathbf{y}_{k+1}\right)^{\sqrt{2}}$

Using DMS, problem (3) can be casted into

$$
\begin{aligned}
\left\{\mathbf{X}^{*}, \mathbf{Y}^{*}\right\}=\min _{\mathbf{X}, \mathbf{Y}} J[\mathbf{X}, \mathbf{Y}] & =\sum_{k \in \mathcal{K}} \mathcal{P}_{k} l(\mathbf{x}, \mathbf{y}) \\
\text { s.t. } \mathbf{h}_{0}\left(\mathbf{x}_{0}, \mathbf{x}_{f}\right) & =0 \\
\mathbf{g}\left(\mathbf{x}_{k}, \mathbf{y}_{k}\right) & \leq 0, k \in \mathcal{K} \cup\{N\} \\
\mathbf{x}_{k+1}-\mathbf{x}_{k}-\mathcal{P}_{k} \mathbf{f}(\mathbf{x}, \mathbf{y}) & =0, k \in \mathcal{K}
\end{aligned}
$$

Iterating a solution of this problem using a nonlinear programmer is referred to as running the trajectory optimizer.

W.r.t. (3), we may still choose variable $\mathbf{y}$ to represent control force, $\mathbf{u}$, either let it represent acceleration in the generalised coordinate space, $\ddot{\mathbf{q}}$. Although equivalent in terms of the formal solution of problem (3), this choice has a profound effect regarding numerical solution of (4).

Forward convention Conventionally, $\mathbf{y}$ is chosen equal to the control force, $\mathbf{u}$, so that functions $\mathbf{f}$ and $l$ have arguments $(\mathbf{x}, \mathbf{u})$. The dependent physical quantity is in this case $\dot{\mathbf{v}}$ so that a forward dynamic model representation is required. Then state-space model, $\mathbf{f}$, and hence the constraint function of problem (4), will depend explicitly on the approximate set, $\hat{\mathcal{S}}$. Any approximate set updates would affect the nonlinear constraint function.

Inverse convention For the second case, we choose $\mathbf{y}=$ $\ddot{\mathbf{q}}$, and so functions $\mathbf{f}$ and $l$ have arguments $(\mathbf{x}, \ddot{\mathbf{q}})$. The dependent physical quantity now equals $\tau$ and an inverse dynamic model representation is requirerd. One

\footnotetext{
${ }^{2}$ Due to the fact that the optimization algorithm is inherently iterative, we can use an implicit integration scheme without stalling solution time.
} 


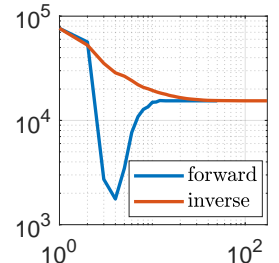

(a) cost

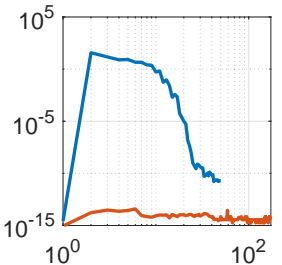

(b) $\|$ constraint $\|$

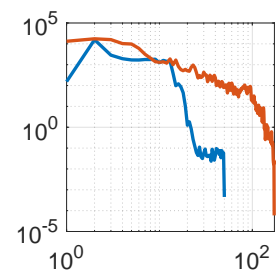

(c) \|f.o.o. $\|$
Fig. 2: Convergence of the optimizer for forward and inverse convention with exact dynamics (DOP from section IV).

may note that in this case the state-space function, $\mathbf{f}$, is linear in it arguments and that $l$ will depend on the approximate set $\hat{\mathcal{S}}$. Any approximate set updates would now affect the nonlinear cost function.

Corresponding the optimization convention that is adopted, the AADO algorithm will thus either act on the cost function be it on the constraint function of problem (4).

We remark that the use of the forward and inverse conventions will affect the convergence of the nonlinear programmer. As an illustration we refer to Fig. 2 where we have depicted the numerical convergence of the trajectory optimizer ${ }^{3}$ in both the forward and inverse convention. The results are for the DOP from the case study in section IV] and we initialize the programmer with a feasible solution. Due to the fact that in the inverse convention the constraint function is linear in its arguments, the optimizer directly changes to the fixed subspace spanned by these linear constraints and hence remains feasible during the entire optimization process. This is not the case in the forward setting since then the constrained function is nonlinear so that the linearized solution subspace chances in between every iteration. This difference is crucial and explains how the solution space is explored in both conventions. Clearly in the forward convention the solution space is first explored towards regions that have minimal control cost. From this region the optimizer iterates towards a solution that is also feasible. The inverse alternative slowly pushes the solution towards a minimal cost. Because that the solution is feasible in each iteration, the low control cost region is never explored. Ultimately we note that in the forward convention the number of iterations may be less but the total number of function evaluations is about the same. This is because the forward convention requires more function evaluations during the line search.

\section{THE AADO ALGORITHM}

The architecture of AADO is based on derivative-free optimizers (DFO) for nonlinear objective functions [11].

AADO iterates over the following steps

step 0 generate initial sample set $\mathcal{X}_{0}$ and evaluate $\mathcal{S}\left(\mathcal{X}_{0}\right)$

step 1 execute $L$ trajectory iterations with the trajectory optimizer whilst using approximate set, $\hat{\mathcal{S}}_{i}$ based on $\left\{\mathcal{X}_{0}, \mathcal{S}\left(\mathcal{X}_{0}\right)\right\}$, identifying candidate solution, $\left\{\mathbf{X}_{i}, \mathbf{Y}_{i}\right\}$

step 2 call the trajectory sampling scheme to generate a set $\mathcal{X}_{i}^{+}$and evaluate approximate set update $\mathcal{S}\left(\mathcal{X}_{i}^{+}\right)$

step 3 evaluate and verify stopping condition, $\rho_{i}>\rho$ based on the update $\mathcal{S}\left(\mathcal{X}_{i}^{+}\right)$, whether to accept the candidate solution, if not update the sets and proceed with step 1

${ }^{3}$ Here $\|$ foo $\|$ stands for the norm of the first order optimality conditions.

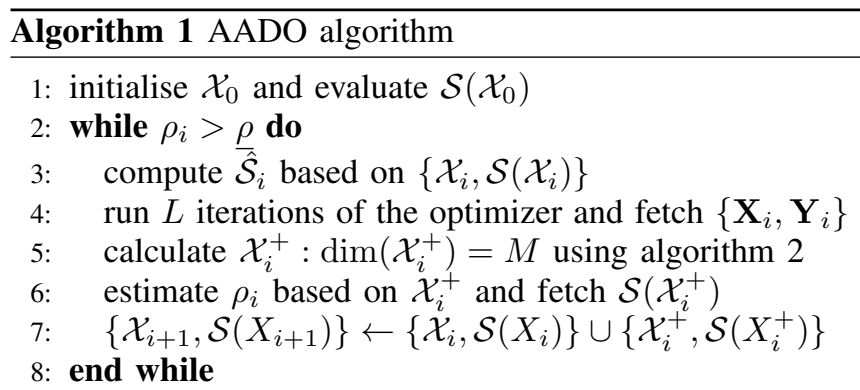

Pseudocode for AADO is given in algorithm 1. One may note that the major feature exhibited by the algorithm is the trajectory sampling, considering that both the stopping condition as the approximate set update depend on it.

The fact that we can use the same set, $\mathcal{X}_{i}^{+}$, both to verify the stopping condition as to refine the approximate model set, is justified due to the fact that the optimization variables in (4) inherently span a subregion of the feasible modeling space instead of a single point. In DFO, one evaluates the high-fidelity set, $\mathcal{S}$, at the current solution and the next action is based on how well $\mathcal{S}\left(\mathbf{X}_{i}\right)$ is predicted by $\hat{\mathcal{S}}_{i}\left(\mathbf{X}_{i}\right)$. The goal of the stopping condition is simply to verify whether the current approximate set $\hat{\mathcal{S}}_{i}$ is sufficiently accurate w.r.t. the current solution. Next the required evaluations $\mathcal{S}\left(\mathbf{X}_{i}\right)$ are extended with explorative samples and are then used to update $\hat{\mathcal{S}}_{i}$. In our framework however, evaluating the approximate set at every point corresponding the current snapshots, $\mathbf{X}_{i}$, would require too many high-fidelity evaluations and would result in overfitting of the function approximator. As such we take a reduced number of samples, that by clever sampling are still able to represent the current solution. Moreover since these samples span the feasible subspace by construction and that $\mathcal{S}$ is evaluated at these samples to compute $\rho_{i}$, we propose simply to recycle $\mathcal{X}_{i}^{+}$to update $\hat{\mathcal{S}}_{i}$.

\section{A. Trajectory sampling scheme}

We postulate the following dynamic stopping criteria, $\rho$, which is a natural metric for the dynamic mismatch introduced by $\hat{\mathcal{S}}_{i}$ w.r.t. $\mathcal{S}$. Exact calculation of this indicator is not desirable given the large amount of high-fidelity function evaluations that would be required. Therefore it is proposed to approximate $\rho$ using a coarse sample average at selected sample instants, $t_{j} \in \mathcal{A}_{i}$

$$
\begin{aligned}
\rho\left[\mathbf{X}_{i}, \mathbf{Y}_{i}\right] & =\frac{1}{T} \int_{\mathcal{T}}\|\mathbf{f}-\hat{\mathbf{f}}\|^{2} \mathrm{~d} t \\
& \approx \frac{1}{N_{s}} \sum_{t_{j} \in \mathcal{A}_{i}}\left\|\mathbf{f}\left(t_{j}\right)-\hat{\mathbf{f}}\left(t_{j}\right)\right\|^{2}
\end{aligned}
$$

The sampling scheme is formalized in Algorithm 2 The main idea is to postulate a set of time instants $\mathcal{C}$, that is e.g. uniform, and to add desirable characteristics to this sample set by application of nonlinear mappings, $m$. In particular, we will consider two inverse maps, namely $b_{i}^{-1}$ and $c_{i}^{-1}$, that is $\mathcal{A} \stackrel{b_{i}^{-1}}{\longleftarrow} \mathcal{B} \stackrel{c_{i}^{-1}}{\longleftarrow} \mathcal{C}$. We construct the maps in a backward fashion as their construction is more intuitive that way. Note that AADO will use forward application of the maps. 


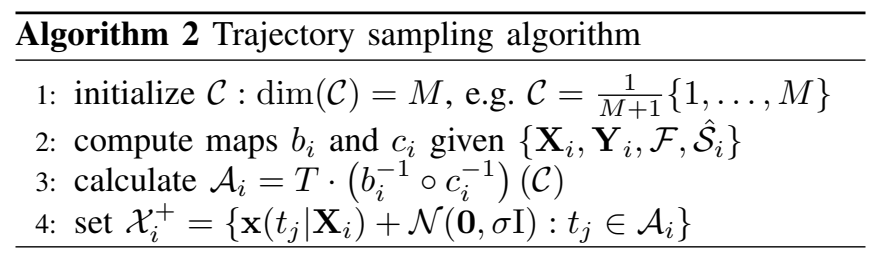

The first map accounts for the fact that a uniform set in the time domain does not correspond with a uniform set in the spatial modeling domain. Therefore, $b_{i}$ maps samples taken into the time domain to samples taken as a function of the (normalized) arc length, $\tau$, along trajectory, $\mathbf{x}\left(\cdot \mid \mathbf{X}_{i}\right)$. Here $\mathbf{x}(t \mid \mathbf{X})$ represents the state value at time instant $t$ based on shooting nodes $\mathbf{X}$ using some interpolation method.

Accordingly, we have

$$
b(t)=\int_{0}^{t} \frac{\mathrm{d} b}{\mathrm{~d} t}\left(t^{\prime}\right) \mathrm{d} t^{\prime} \leftarrow \frac{\mathrm{d} b}{\mathrm{~d} t}(t)=\left\|\frac{\mathrm{d} \mathbf{x}}{\mathrm{d} t}\right\|_{2}=\|\mathbf{f}(t)\|_{2}
$$

The second map accounts for the fact that the previous sample set $\mathcal{X}_{i}$ may be locally sparse. Inspired by the previous mapping, we can construct a map so that spatially uniform samples along the trajectory will gravitate towards regions that are sparsely sampled by the current set $\mathcal{X}_{i}$.

To accomplish this, we require a measure for the local sample sparsity conditional on the set $\mathcal{X}_{i}$ as a function of trajectory arc length, say $p(\tau)=p\left(\mathbf{x}\left(\tau \mid \mathbf{X}_{i}\right) \mid \mathcal{X}_{i}\right)$.

$$
c(\tau)=\int_{0}^{\tau} \frac{\mathrm{d} c}{\mathrm{~d} \tau}\left(\tau^{\prime}\right) \mathrm{d} \tau^{\prime} \leftarrow \frac{\mathrm{d} c}{\mathrm{~d} \tau}(\tau)=p(\tau)
$$

A desirable sample set, $\mathcal{A}_{i}$, in the time domain is then obtained as illustrated in the trajectory sampling algorithm. In conclusion we add a small gaussian noise term, so to break any emergent symmetries and in order to assist the algorithm in exploring the modeling space, so that the eventual update set $\mathcal{X}_{i}^{+}=\left\{\mathbf{x}\left(t_{j} \mid \mathbf{X}_{i}\right)+\mathcal{N}(\mathbf{0}, \sigma \mathrm{I}): t_{j} \in \mathcal{A}_{i}\right\}$.

This framework gives rise to different trajectory sampling strategies, as are demonstrated in Fig. 3

\section{B. Sources of stochasticity}

We note that no two runs of the algorithm produce the exact same sequence of intermediate results. First of all the algorithm needs to be initialized with a certain sample set $\mathcal{X}_{0}$. Here we adopted a Latin Hypercube Sampling (LHS) [12]. We emphasize that it is crucial to generate an initial LHS, $\mathcal{X}_{0}$, that spans the entire solution space such that a general notion of the dynamic landscape is obtained. Therefore the update sets, $\mathcal{X}_{i}^{+}$, can focus on exploitation rather than on shear exploration. That is because the dynamic landscape has been explored in any direction of the feasbile solution space by the LHS. Secondly, one may consider the small Gaussian noise term that is added to the update sets. Especially in the final stage of the algorithm, this small gaussian step is crucial to obtain a more robust load model gradient estimate in the neighbourhood of the final trajectory. Otherwise the local spatial coverage of the samples, normal w.r.t. to the trajectory from which they are drawn, is only guaranteed by the spatial shift in between consecutive trajectories.

\section{A function approximator: the Kriging predictor}

Several general nonlinear function approximators can be considered to fit an approximate model set, $\hat{\mathcal{S}}_{i}$, to sample

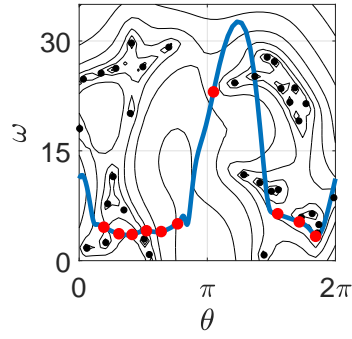

(a) equitemporal sampling, i.e. $\mathcal{C}$

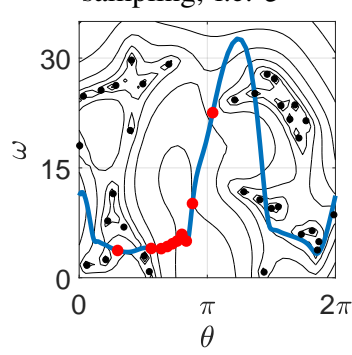

(c) improbable equitemporal sampling, i.e. $c_{i}^{-1}(\mathcal{C})$

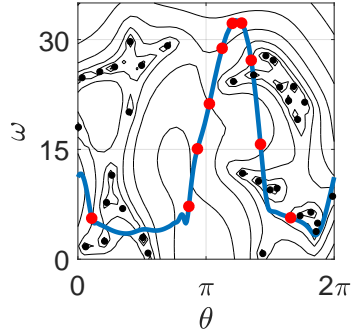

(b) equidistant sampling,

i.e. $b_{i}^{-1}(\mathcal{C})$

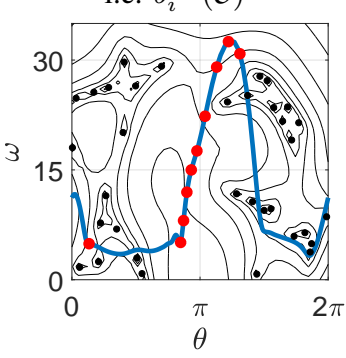

(d) improbable equidistant sampling, i.e.
Fig. 3: Comparison of adaptive sample schemes for sampling along the current trajectory. Black markers indicate previous samples, $\mathcal{X}$, whilst red markers indicate the set update, $\mathcal{X}^{+}$. The background contour visualizes $\log p(\mathbf{x} \mid \mathcal{X})$.

data $\left\{\mathcal{X}_{i}, \mathcal{S}\left(\mathcal{X}_{i}\right)\right\}$. In this work we considered the Kriging predictor due to the natural sparsity measure that is included in this framework. The Kriging predictor is derived from assumption (6) (here the output dimension equals 1 for simplicity, generalisation is trivial). For details see [6].

Here $\mathbf{p}(\mathbf{x})$ is a vector function containing a number of regression functions, e.g. linear, quadratic, etc. $z(\mathbf{x})$ is an unbiased random process for which the output values $z\left(\mathbf{x}_{i}\right)$ and $z\left(\mathbf{x}_{j}\right)$ are spatially correlated, i.e. we model $\operatorname{cov}\left(z_{i}, z_{j}\right)$ using a radial basis function, $\operatorname{cov}\left(z_{i}, z_{j}\right)=\sigma^{2} r\left(d\left(\mathbf{x}_{i}, \mathbf{x}_{j}\right)\right)$.

$$
\mathcal{S}(\mathbf{x})=\mathbf{p}(\mathbf{x})^{\top} \mathbf{c}+z(\mathbf{x})
$$

From above made assumptions, the Kriging framework derives the most likely linear predictor, given by

$$
\hat{\mathcal{S}}(\mathbf{x})=\mathbf{p}(\mathbf{x})^{\top} \mathbf{c}+\mathbf{r}(\mathbf{x})^{\top} \mathbf{d}
$$

Here $\mathbf{r}(\mathbf{x})$ is a vector function which entries are determined by, $r\left(d\left(\mathbf{x}, \mathbf{x}_{j} \mid \boldsymbol{\theta}\right)\right), \mathbf{x}_{j} \in \mathcal{X}_{i}$ where $\boldsymbol{\theta}$ is a hyperparameter that affects distance, $d$, usually $d\left(\mathbf{x}_{i}, \mathbf{x}_{j} \mid \boldsymbol{\theta}\right)=\left\|\mathbf{x}_{i}-\mathbf{x}_{j}\right\|_{\boldsymbol{\theta}}$. $\mathbf{c}$ and $\mathbf{d}$ are coefficient vectors that are both linear in $\mathcal{S}\left(\mathcal{X}_{i}\right)$ (represented as a vector). Due to the stochastic preconditions, we can calculate the mean squared error of the predictor by means of (7). This function is a natural measure for the conditional sampling sparsity.

$$
p\left(\mathbf{x} \mid \mathcal{X}_{i}\right)=\sigma^{2}\left(1-\mathbf{r}^{\top} \mathrm{R}^{-1} \mathbf{r}+\tilde{\mathbf{r}}^{\top}\left(\mathrm{P}^{\top} \mathrm{R}^{-1} \mathrm{P}\right)^{-1} \tilde{\mathbf{r}}\right)
$$

where $\tilde{\mathbf{r}}=\mathrm{P}^{\top} \mathrm{R}^{-1} \mathbf{r}-\mathbf{p}$ and where the capital variables represent Vandermonde matrices of the sample set $\mathcal{X}_{i}$ for the corresponding lowercase vector functions. Note that the expression is independent of sampled function values, $\mathcal{S}\left(\mathcal{X}_{i}\right)$. 
TABLE I: Lumped model parameters of the slider-crank rigid body model.

\begin{tabular}{ccccccccc}
$l_{1}$ & $l_{2}$ & $m_{1}$ & $m_{2}$ & $m_{3}$ & $J_{1}$ & $J_{2}$ & $J_{m}$ & $B_{m}$ \\
\hline 0.4 & 0.6 & 0.5 & 0.5 & 0.6 & 0.0067 & 0.015 & 0.0025 & 0.01
\end{tabular}

\section{Technical notes on the implementation}

We finalise this section with a short note on some technical details of our implementation. We use an in-house implementation of the DMS transcription method and the SQP setting from the build-in MATLAB function fmincon. This functions uses the BFGS algorithm to update the Hessian of nonlinear program (4). Due to the fact that once we have updated the set, $\mathcal{S}$, the gradient landscape in the neighbourhood of the current optimization variable value differs significantly from the previous landscape, can cause break down of the BFGS update ultimately causing premature stop of the optimizer. This phenomenon has been observed in our numerical experiments and could succesfully be alleviated by reinitializing the optimizer entirely. We have used the DACE-toolbox to obtain a Kriging predictor for given sample set $\left\{\mathcal{X}_{i}, \mathcal{S}\left(\mathcal{X}_{i}\right)\right\}$ [6]. In conclusion, abitrary instants $\mathbf{x}\left(t \mid \mathbf{X}_{i}\right)$ were evaluated using spline interpolation in between snapshot couples, $\left\{\mathbf{t}, \mathbf{X}_{i}\right\}$.

\section{CASE STUdY : SLIDER-CRANK}

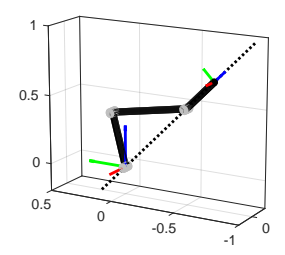

Fig. 4: Slidercrank model configuration
In this section we apply the AADO algorithm on a tilted slider-crank (Fig. 4) that is modeled using Newton-Euler equations of motion that are fast $(\mathcal{F})$ together with a load model that represents the slow $(\mathcal{S})$ type. We first consider the algorithm used in the forward convention to validate the method against a conventional dynamic optimization using exact dynamics. In a next stage we explore the influence of the DOP convention (forward/inverse) in combination with the different trajectory sampling schemes.

\section{A. Limit cycle dynamic optimization problem}

The rigid multibody dynamics of the slider-crank system can be modelled as a serial linkage of three rods with lengths $l_{1}, l_{2}$ and $l_{3}$ and masses $m_{1}, m_{2}$ and $m_{3}$ respectively. We constraint the linkage point in between rods 2 and 3 to describe a linear motion tilted over an angle $\frac{\pi}{4}$. The third rod is considered to describe a linear motion along the same line so that we do not require knowledge of its planar inertia. The planar inertias of the first two links are denoted as $J_{1}$ and $J_{2}$. We further consider a small motor intertia, $J_{m}$, and viscous friction, $B_{m}$. Lumped model parameter values used in this study are given in Table I].

For demonstrational purposes we adopted an analytical nonlinear load model $w$. Here $x$ and $v$ are functions that express the linear position and velocity w.r.t. the origin.

$$
w(\mathbf{x})=2.5\left(1+3\left|x(\mathbf{x})-l_{2}\right|^{1.6}\right) \operatorname{sgn}(v(\mathbf{x}))|v(\mathbf{x})|^{2.3} \frac{\sqrt{2}}{2} m_{3} g
$$

Our goal is to calculate an energy optimal limit cycle for following cost rate function $l(\mathbf{x}, \mathbf{u})=\dot{\theta} u+v(\mathbf{x})^{2}+u^{2}$. In conclusion we fix the limit cycle period to $T=1 \mathrm{~s}$.

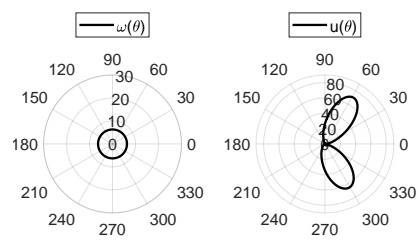

(a) nominal cycle

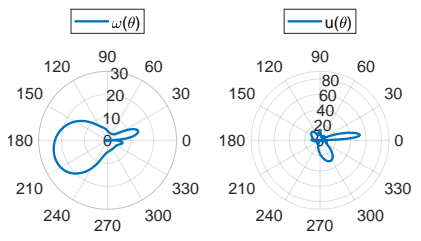
mate load model using 120 latin hypercube samples (c) optimal cycle with approxi-
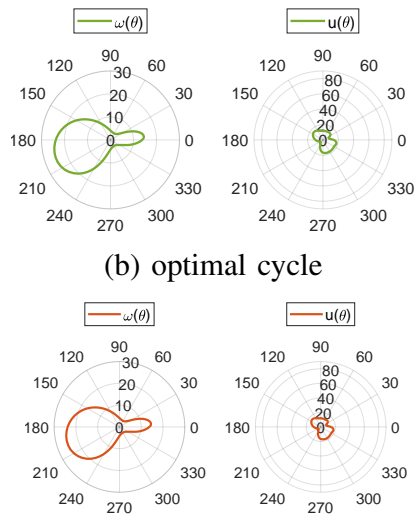

(d) optimal cycle with adaptive approximate load model using 120 samples in total (b) optimal cycle

Fig. 5: Comparison of slider-crank cycles for the academic load model. A visually equivalent optimal solution is found using the adaptive approximate model.

We discretise problem (3) with 55 snapshots and initialize the solver with the nominal cycle, i.e. a constant speed cycle with corresponding control forces computed using the inverse dynamic model and approximate load model. Problem (4) was implemented and solved as described in section III-D

\section{B. Approximate versus adaptive approximate}

We start our numerical demonstration with a comparison between the approximate (Fig. 1p) and adaptive approximate (Fig. 1R) dynamic optimization algorithms. In each scenario, load model $w$ was approximated as a function of the generalised coordinate space 4 . We used the forward convention for both solution methods. AADO was initialized using a LHS $\mathcal{X}_{0} \subset[0,2 \pi] \times[0,35]$ with $\left|\mathcal{X}_{0}\right|=K=50$ and algorithmic hyperparameter set $(L, M, \sigma, \rho)=\left(15,10,10^{-1}, 1\right)$. For given conditions the AADO algorithm converged after adding 70 adaptive samples. Then we extended the initial LHS of 50 to a set $|\tilde{\mathcal{X}}|=120$. From extended set $\tilde{\mathcal{X}}$, we generated a globally approximate load model which we then used to complete an approximate dynamic optimization for comparison with AADO.

In Fig. 5, we compare the nominal constant speed cycle with control force computed using the inverse dynamic model and exact load model, $w$, with optimal cycles obtained by solving (4) for given optimality definitions with different algorithms. The accumulated costs equal $7.64 \times 10^{4} \mathrm{~J}$ for the nominal cycle, and, $1.54 \times 10^{4} \mathrm{~J}$ for the (correct) optimal cycles. The most important observation is that a visually equivalent optimal cycle is obtained using AADO, which used only 120 load model evaluations in comparison to the 29.480(!) evaluations for computing the exact solution. Note that the load model gradients were calculated using forward finite differencing. The globally approximate load model clearly misjudges the dynamic landscape and an infeasible solution (w.r.t. the true dynamics) is retrieved. In Fig. 6 we visualize the load model approximation accuracy along optimal trajectory, $\mathbf{x}^{*}$. By comparing function values, we can judge the true physical feasibility, whilst by comparing

\footnotetext{
${ }^{4}$ Alternatively, the load model can be approximated w.r.t. to the (local) contact space coordinates to avoid nonlinearities introduced by the forward kinematic model.
} 

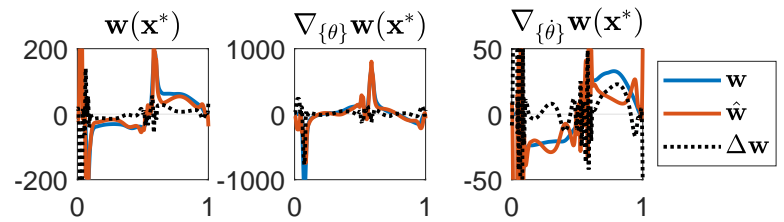

(a) approximate load model using 120 latin hypercube samples
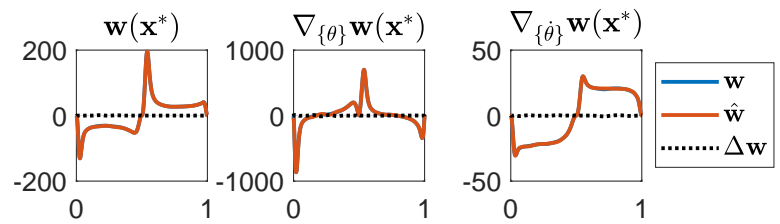

(b) adaptive approximate load model using 120 samples in total

Fig. 6: Comparison between function evaluations and derivatives along optimal solution.

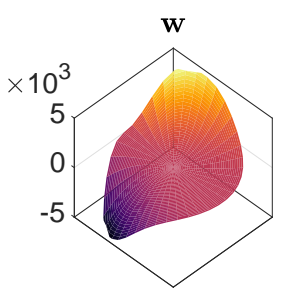

(a) exact load model
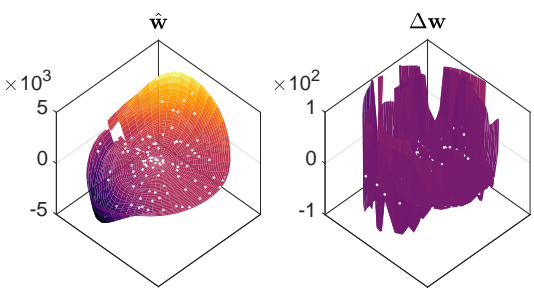

(b) approximate load model using 120 latin hypercube samples
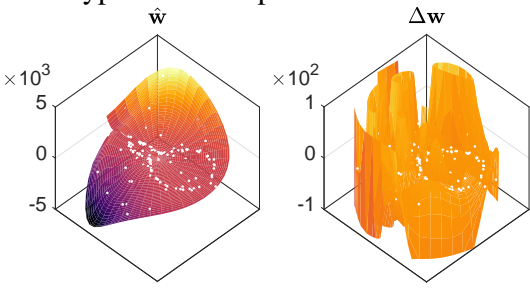

(c) adaptive approximate load model using 120 samples in total

Fig. 7: Comparison between exact load model surface (polar grid as in Fig. 5) and approximate load surfaces using Kriging. Sample points are indicated with the white markers.

the derivative values, we can judge the local first order optimality of the final trajectories and corresponding final approximate load model. Clearly the AADO algorithm has generated an approximate load model that is first order exact at the final solution. Although the globally approximate load model emulates the true load model reasonably accurate, the local derivative evaluations deviate strongly from their true value and therefore the 1 st order optimality is misjudged. Conclusively, one can inspect the global surface approximations in Fig. 7 where the error of both the adaptive as the global load models are similar in magnitude. The adaptive load model is however exact in the neighbourhood of the final trajectory.

\section{Forward versus inverse optimization convention}

The AADO algorithm was originally developed for application in the forward convention [1]. However, the framework does lend itself for application in an inverse setting as well. As was noted in the previous section, the algorithm then no longer approximates the constraint function but facilitates updates of the objective function at every iteration.
Recall that in the inverse framework the constraint function becomes linear in its arguments and does not depend on the high-fidelity load model. Hence each solution generated by the trajectory optimizer will be exact w.r.t. f. As a result we can not use the same dynamic stopping criteria. Adhering the inverse convention, the dynamic feasibility of the load model approximation can not be estimated directly. We propose therefore to change the integrand of (5) for $\|l-\hat{l}\|^{2}$. Transition from the forward to the inverse convention does not require to alter the sampling strategy since that the characteristics of the mappings can be maintained. That is, f still equals $\dot{\mathbf{x}}$ and $p$ only depends on the local sampling sparsity. Note however that because now $\mathbf{f}$ is linear, hence each iteration of the trajectory optimizer is feasible. We will thus have an exact estimate of $\dot{\mathbf{x}}$. This was not the case in the forward convention (as can be seen in Fig. 3), where (b) does not perfectly distribute the points equidistantly along the trajectory since here the solution may be infeasible.

Fig. 8 portrays convergence curves for both the forward and the inverse convention and compares the different sampling strategies. Following the described stochasticity of the framework, each setting (that is according the sampling strategy and DOP convention) of the algorithm was executed 25 times and averaged to indicate the resulting performance and robustness. The hyperparameter set was fixed for all experiments, $(K, L, M, \sigma)=\left(60,15,15,10^{-1}\right)$. We used following metrics for comparison. The relative cost function value, the constraint function evaluated with the exact dynamics, evolution of the dynamic stopping criteria and the number of sub-iterations after which the trajectory optimizer converged (the upper limit is fixed by the framework but it is possible that the trajectory optimizer requires less iterations to converge, certainly when the outer loop starts to converge).

Following remarks can be made. In the forward convention the sampling schemes that do not use the local sparsity measure, $p$, clearly underperform. That is because there is no feedback between earlier samples updates and the new sample update. Hence as the solution converges, the generated sample set converges and the only exploration is facilitated by the small gaussian noise term. To our surprise, the constraint value (in the forward convention a direct measure for the load model approximation) converges faster using the improbable equitemporal strategy (i.e. $c_{i}^{-1}$ ). We argue that this is because more samples are generated in regions where the trajectory crosses the state space at a slow pace. Hence these regions will have a larger contribution to the constraint functions since the discretisation time grid is also equitemporal. Further one may note that all sample strategies have a similar convergence rate w.r.t. the stopping criteria. Clearly the equidistant and -temporal strategies greatly underestimate the actual dynamic error. In conclusion we note that although the average convergence of the improbable equitemporal strategy outperforms the other strategies, the improbable equidistant strategy is more robust.

Considering the inverse DOP convention, the results are less clear on what sampling strategy is more optimal. In any case the cost function converges to a local minimal limit cycle, that although feasible, has a larger energy content than the true solution recovered by the forward convention. This is because the trajectory optimizer is operating in a feasible linear subspace and pushes the solution towards a 

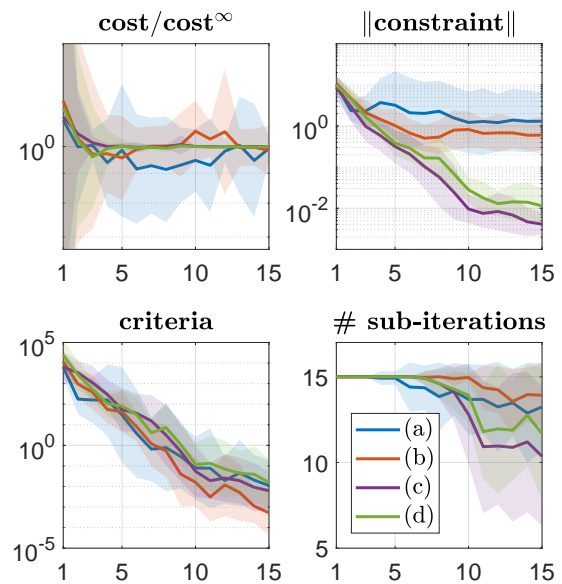

(a) forward convention,

$\rho_{i} \propto \int\|\mathbf{f}-\hat{\mathbf{f}}\|^{2} \mathrm{~d} t$
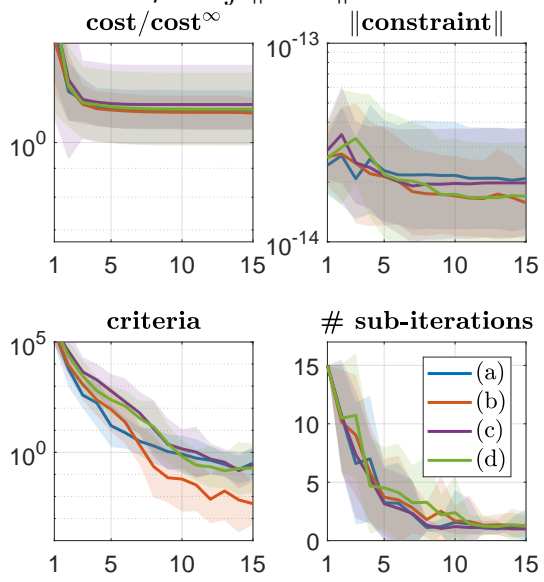

(b) inverse convention, $\rho_{i} \propto \int\|l-\hat{l}\|^{2} \mathrm{~d} t$

Fig. 8: Visualization of AADO convergence for different sampling schemes and optimization convention with hyperparameter set $(K, L, M, \sigma)=\left(60,15,15,10^{-1}\right)$. The legend refers to the different sample schemes as defined in Fig. 3 .

region with low cost. In the initial AADO iterations the solution hence settles for a local minima respective to the approximate load model. After a model update, the trajectory optimizer cannot recover from this initial local solution and converges to locally feasible but suboptimal solution. This also explains the wide uncertainty interval around the final cost function value. Due to above described mechanism, the sampling strategy has a lesser decisive influence.

\section{CONCLUSiON}

In this work we present the Adaptive Approximate Dynamic Optimization algorithm. The AADO algorithm is tailored to solve dynamic optimization problems where the dynamic model functions can be divided into a subset containing model parts that can be evaluated with low computational cost and a subset that contains high-fidelity (i.e. computationally costly) model parts that delay the solution.

To accomodate this issue the AADO algorithm engages an approximate high-fidelity model set. The approximate highfidelity set is generated using a general function approximator and a set of high-fidelity model samples. The AADO algorithm iterates between the trajectory optimizer and the function approximator and uses the trajectory optimizer as guidance to focus the sample effort to the neighbourhood of the true solution. The samples are generated using an original trajectory sampling algorithm.

The AADO algorithm was originally developed for application in a forward dynamic optimization convention. In this work we extend the AADO algorithm's application to inverse dynamic optimizaton settings. The influence of the dynamic optimization convention and the use of different sampling strategies is explored and discussed numerically on the basis of a slider-crank case study.

We found that the AADO algorithm requires only $0.1 \%$ system model evaluations compared with conventional DO and only $30 \%$ compared with non-adaptive AADO. Our results consolidate the methods potential and invite for further research.

\section{REFERENCES}

[1] T. Lefebvre, F. De Belie, and G. Crevecoeur. A trajectory-based sampling strategy for sequentially refined metamodel management of metamodel-based dynamic optimization in mechatronics. Optimal Control Applications and Methods, 2018.

[2] A. Deshmukh and J. Allison. Design of nonlinear dynamic systems using surrogate models of derivative functions. Journal of Mechanical Design, 139(10):101402-1011402-12, 2017.

[3] R. Lin, Y.and Haftka, N. Queipo, and B. Fregly. Surrogate articular contact models for computationally efficient multibody dynamic simulations. Medical engineering \& physics, 32(6):584-594, 2010.

[4] J. Halloran, A. Erdemir, and A. Van Den Bogert. Adaptive surrogate modeling for efficient coupling of musculoskeletal control and tissue deformation models. Journal of biomechanical engineering, 131(1):011014, 2009.

[5] L. Magnier and F. Haghighat. Multiobjective optimization of building design using trnsys simulations, genetic algorithm, and artificial neural network. Building and Environment, 45(3):739-746, 2010.

[6] S. Lophaven, H. Nielsen, and J. Søndergaard. DACE - a matlab kriging toolbox, version 2.0. Technical report, Informatics and Mathematical Modelling, Technical University of Denmark, DTU, Richard Petersens Plads, Building 321, DK-2800 Kgs. Lyngby, 2002.

[7] K. Crombecq, D. Gorissen, D. Deschrijver, and T. Dhaene. A novel hybrid sequential design strategy for global surrogate modeling of computer experiments. SIAM Journal on Scientific Computing, 33(4):1948-1974, 2011.

[8] J. van der Herten, I. Couckuyt, D. Deschrijver, and T. Dhaene. A fuzzy hybrid sequential design strategy for global surrogate modeling of high-dimensional computer experiments. SIAM Journal on Scientific Computing, 37(2):A1020-A1039, 2015.

[9] M. Diehl, H. G. Bock, H. Diedam, and P. B. Wieber. Fast direct multiple shooting algorithms for optimal robot control. In Fast motions in biomechanics and robotics, pages 65-93. Springer, 2006.

[10] T. Erez and E. Todorov. Trajectory optimization for domains with contacts using inverse dynamics. In Intelligent Robots and Systems (IROS), 2012 IEEE/RSJ International Conference on, pages 49144919. IEEE, 2012.

[11] L. Rios and N. Sahinidis. Derivative-free optimization: a review of algorithms and comparison of software implementations. Journal of Global Optimization, 56(3):1247-1293, 2013.

[12] J. Sacks, W. J. Welch, T. J. Mitchell, and H. P. Wynn. Design and analysis of computer experiments. Statistical science, pages 409-423, 1989. 